\title{
Multicenter, Open-Label, Phase I Study of DSP-7888 Dosing Emulsion in Patients with Advanced Malignancies
}

\author{
Alexander Spira ${ }^{1,2}$ (1) $\cdot$ Aaron R. Hansen ${ }^{3} \cdot$ Wael A. Harb ${ }^{4} \cdot$ Kelly K. Curtis ${ }^{5} \cdot$ Erina Koga-Yamakawa $^{6}$. \\ Makoto Origuchi ${ }^{7} \cdot$ Zhonggai Li $^{8} \cdot$ Bella Ertik $^{9} \cdot$ Walid L. Shaib ${ }^{10}$
}

Accepted: 2 April 2021 / Published online: 3 May 2021

(c) The Author(s) 2021

\begin{abstract}
Background Wilms' tumor 1 (WT1) is overexpressed in various malignancies. DSP-7888 Dosing Emulsion, also known as ombipepimut-S (United States Adopted Name; International Nonproprietary Name: adegramotide/nelatimotide), is an investigational therapeutic cancer vaccine comprising two synthetic peptides derived from WT1 to promote both cytotoxic T-lymphocyte (CTL) and helper T-lymphocyte-mediated immune responses against $W T 1$-expressing tumors.

Objective The aim of this study was to report the results from a phase I dose-escalation study (NCT02498665) that evaluated DSP-7888, administered either intradermally (ID) or subcutaneously (SC), in patients with recurrent or advanced malignancies associated with overexpression of WTI.

Patients and Methods In this phase I dose-escalation study, patients with recurrent or advanced malignancies associated with overexpression of WTl who progressed on, were intolerant to, or not a candidate for standard therapy or who presented with a malignancy that had no definite standard therapy received escalating doses of ID or SC DSP-7888 in a rolling-six study design. DSP-7888 3.5, 10.5, or 17.5 (ID only) mg was administered until disease progression or other discontinuation event. Primary objectives were safety, tolerability, and identification of the recommended phase II dose (RP2D). Overall survival (OS) and WT1-specific CTL induction were included as secondary and exploratory objectives, respectively.

Results Twenty-four patients received either ID (3.5 mg, $n=4 ; 10.5 \mathrm{mg}, n=3 ; 17.5 \mathrm{mg}, n=3)$ or SC DSP-7888 (3.5 mg, $n=9 ; 10.5 \mathrm{mg}, n=5)$. No dose-limiting toxicity was observed. The most frequent treatment-emergent adverse event was injection site reactions (ID, 100\% [10/10]; SC, 35.7\% [5/14]); all were grade 1 or 2. Four patients (ID $17.5 \mathrm{mg}, n=1$; SC $3.5 \mathrm{mg}, n=1$; SC $10.5 \mathrm{mg}, n=2)$ had stable disease, 16 had progressive disease, and four were not evaluable. Median (95\% confidence interval) OS duration was 180.0 (136.0-494.0) days. Among evaluable patients, WT1-specific CTL induction was observed in $66.7 \%$ (6/9) and 41.7\% (5/12) of those administered ID and SC DSP-7888, respectively.

Conclusions DSP-7888 Dosing Emulsion was well tolerated, with no dose-limiting toxicities, in patients with recurrent or advanced malignancies. Higher WT1-specific CTL induction activity was noted with ID compared with SC administration; because of this, the ID route was selected for further evaluation in the clinical program.
\end{abstract}

Trial registration ClinicalTrials.gov identifier: NCT02498665.

\section{Background}

Expression of the wild-type Wilms' tumor 1 (WT1) gene has been detected in various hematologic malignancies (e.g., acute myeloid leukemia [AML], acute lymphoid leukemia, myelodysplastic syndrome [MDS]) and solid tumors (e.g., breast, colorectal, glioblastoma, lung, pancreatic) [1-5]. The prevalence of WT1 expression in various cancers ranges from 3 to $91 \%$, with high rates reported in several hematologic and solid cancers, including ovarian, colorectal, AML, and MDS [6-10]. WT1

Alexander Spira

alexander.spira@usoncology.com

Extended author information available on the last page of the article was originally thought to function as a tumor suppressor [11], but later studies uncovered a correlation between its expression and disease relapse/poor prognosis in a variety of malignancies [6, 12-14]. In addition, anti-sense oligomers were shown to inhibit the growth of $W T 1$-expressing cancer cells [15-17]. Based on these findings, $W T l$ has been redefined as an oncogenic driver [15]. This evidence highlights WT1 as a potential therapeutic target for a number of cancers.

Peptide-based vaccines targeting WT1 have been shown to stimulate the production of CD8 + WT1-specific cytotoxic $\mathrm{T}$ lymphocytes (CTLs) and anti-cancer responses in patients with leukemia or solid tumors [18-21]. The WT1 peptidebased vaccines in these studies were designed primarily to 


\section{Key Points}

DSP-7888 Dosing Emulsion is an investigational therapeutic cancer vaccine comprising two synthetic peptides derived from Wilms tumor 1 (WT1) to promote both cytotoxic T-lymphocyte and helper T-lymphocyte-mediated immune responses against WT1-expressing tumors.

DSP-7888, administered either intradermally or subcutaneously, was well tolerated in this phase I study of malignancies. No dose-limiting toxicities were observed.

Based on pharmacodynamic data, intradermal DSP-7888 was selected for further evaluation in advanced solid tumors, including glioblastoma.

elicit WT1-specific CTLs. Optimal anti-tumor responses likely require both WT1-specific CTLs and CD4 + helper T lymphocytes (HTLs) [22, 23]. HTLs play a critical role in anti-cancer responses by promoting dendritic cell presentation of antigen to CTLs, secreting cytokines that maintain CTL proliferation and effector function, and generating CTL memory responses [22-24]. A WT1-specific helper peptide vaccine (OCV-501) has been studied in the clinical setting, but it only induces WT1-specific HTLs (human leukocyte antigen [HLA] class II-restricted), not CTLs [25].

To optimize the potential for anti-cancer activity, the investigational DSP-7888 therapeutic cancer vaccine contains two synthetic peptides derived from WT1 to promote both CTL- and HTL-mediated immune responses against WT1-expressing tumors. The CTL peptide (i.e., the killer peptide) stimulates the generation of CTLs that recognize wild-type WT1 in an HLA-A*02:01-, HLA-A*02:06-, and HLA-A*24:02-restricted manner [26, 27].

We present results from a phase I study (NCT02498665) that evaluated DSP-7888 Dosing Emulsion in patients with recurrent or advanced malignancies associated with overexpression of WT1 who progressed on, were intolerant to, or not a candidate for standard therapy or who presented with a malignancy that had no definite standard therapy. The primary objectives were safety, tolerability, and identification of the recommended phase II dose (RP2D).

\section{Methods}

\subsection{Study Design}

This open-label, dose-escalation, phase I study (NCT02498665) was conducted at eight sites in North America (United States, $n=7$; Canada, $n=1$ ). Patients were assigned to receive escalating doses of intradermal
(ID) or subcutaneous (SC) DSP-7888 3.5, 10.5, or 17.5 (ID only) $\mathrm{mg}$ in a rolling-six study design. Compared with a $3+3$ study design, a rolling-six study design allows dose escalation to proceed more quickly. Unlike a $3+3$ study design, a rolling-six study does not pause each time three patients complete the dose-limiting toxicities (DLT) evaluation period. This design shortens the study conduct timeline compared with a traditional $3+3$ design.

In the present study, patients were first enrolled in the ID group and then the SC group. Initially, three patients were enrolled into a dose cohort (Supplementary Table S1, see electronic supplementary material [ESM]). At the time of enrollment of the fourth study participant, if safety data were available for all three enrolled patients and no DLT had been reported, the fourth patient was enrolled to the next dose level. If data were not available for one or more of the first three enrolled patients, or if a DLT had been observed, this fourth patient was enrolled to the same dose level as the previous three participants. The DLT evaluation period was 28 days. DSP-7888 was administered every week for the first 4 weeks (induction phase), then every 1-2 weeks for 6 weeks (consolidation phase), and every 2-4 weeks thereafter (maintenance phase) until disease progression or other discontinuation criterion was met. The frequency of dosing during the consolidation phase (every week or every 2 weeks) and the maintenance phase (every 2 or every 4 weeks) was based on investigator decision. DSP-7888 was administered as a dosing emulsion (water in oil) containing the adjuvant MONTANIDE ISA 51 VG. The emulsion acts as depot, enhancing the immunogenicity of the two synthetic peptides.

\subsection{Patients}

Eligible participants were aged $\geq 18$ years with histologically or cytologically confirmed advanced malignancies (AML, MDS, glioblastoma multiforme, melanoma, non-small cell lung cancer [NSCLC], ovarian cancer, pancreatic cancer, sarcoma, or renal cell carcinoma), and an Eastern Cooperative Oncology Group performance status of $\leq 2$. Patients with MDS had to have an International Prognostic Scoring System (IPSS) score $\geq 1.5$ or an IPSS score $<1.5$ if transfusion dependent (requiring $\geq 2$ units of red blood cells or $\geq 10$ units of platelets in the 8 weeks prior to enrollment). For complete enrollment criteria, see Supplementary Methods S1 (ESM).

\subsection{Outcomes}

The primary objectives were to determine the safety, tolerability, and RP2D of DSP-7888 in adults with advanced malignancies when administered either ID or SC. The secondary objectives were to assess the preliminary anti-tumor 
activity, overall survival (OS), and progression-free survival (PFS) of DSP-7888 in patients with advanced malignancies and time to transformation to AML or death in patients with MDS. Pharmacodynamics analyses, including the assessment of WT1-specific CTL induction in all study participants and the expression of HLA in patients with solid tumors, were exploratory objectives.

\subsection{Assessments}

Treatment-emergent adverse events (TEAEs), defined as an event post-treatment that was either absent pretreatment or worsened relative to the pretreatment state, were graded with the National Cancer Institute Common Terminology Criteria for Adverse Events (version 4.0). DLTs were defined in the protocol (see Supplementary Methods S2, ESM). The RP2D was defined as the highest tested dose at which DLTs occurred in $\leq 1$ of 6 patients. In the event the RP2D could not be determined, the dose and administration route of DSP-7888 recommended for subsequent studies would be based on a comprehensive review of safety, tolerability, and biomarker information from each cohort.

Anti-tumor activity was assessed every 8 weeks after the first dose of DSP-7888 according to immune-related response criteria [28, 29], Gynecological Cancer Intergroup (GCIG) criteria (ovarian cancer) [30], or International Working Group (IWG) criteria (AML or MDS) [31, 32].

Peripheral blood samples were obtained for the evaluation of WT1-specific CTL induction activity by tetramer assay at weeks $2,4,8,12$, and 16 and on the day of administration of every other dose thereafter. The assay contained three WT1 tetramer types. Two detected WT1-specific CTLs restricted to HLA A02:01 or A02:06, and the third detected WT1-specific CTLs restricted to HLA A24:02. HLA expression was evaluated via immunohistochemistry in patients with solid tumors. Additional methods are provided in Supplementary Methods S3 and S4 (see ESM).

\subsection{Statistics}

The sample size was determined based on clinical rather than statistical considerations. If the true underlying rates of DLTs were $0.1,0.2,0.3,0.4$, and 0.5 , then the chances of dose escalation at a given dose level were $91 \%, 71 \%$, $49 \%, 31 \%$, and $17 \%$, respectively, with six patients per dose cohort. Safety analyses were performed on the safety population (patients who received $\geq 1$ dose of DSP-7888) and summarized using descriptive statistics. Efficacy analyses were performed on the intent-to-treat population, which was composed of all enrolled patients, irrespective of whether they had received study treatment. Best overall response was summarized using descriptive statistics. OS, PFS, and time to transformation to AML or death were estimated using the method of Kaplan-Meier. Statistical analyses were performed using SAS (version 9.3 or higher).

This study was conducted in compliance with the Declaration of Helsinki, the International Conference on Harmonization Guidelines for Good Clinical Practice, and applicable national and local regulatory requirements. The study protocol was approved by the Independent Ethics Committee or Institutional Review Board at each participating site. All patients provided written informed consent prior to study participation.

\section{Results}

\subsection{Patients}

This phase I study was conducted between March 7, 2016, and December 17, 2018, in patients with advanced malignancies who were heavily pretreated. Twenty-four patients were assigned to and received one or more doses of ID DSP-7888 $(n=10)$ or SC DSP-7888 $(n=14)$ (Supplementary Figure S1, see ESM). Most patients were male (62.5\%) and White $(91.7 \%$ ) (Table 1), and had received a median of three (range 1-6) prior lines of therapy. The most frequently reported type of previous cancer therapy was chemotherapy (95.8\%), though patients also received previous immunotherapy (37.5\%), biologic therapy (16.7\%), or treatment with an investigational agent (29.2\%). Among study participants, glioblastoma multiforme (29.2\%) and pancreatic cancer (29.2\%) were the most common tumor types.

The median (range) overall duration of treatment was 8.00 (1.0-49.9) weeks. The dose of DSP-7888 was not reduced in any study participant. All patients $(N=24)$ discontinued DSP-7888 (Supplementary Figure S1, see ESM); the most common reason for discontinuation was disease progression per immune-related response, GCIG, or IWG criteria (15/24 [62.5\%]). Other reasons for discontinuation were study withdrawal $(n=3)$, clinical progression $(n=3)$, investigator decision $(n=2)$, and death $(n=1)$ (Supplementary Figure S1, see ESM); this death was attributable to cardio-respiratory arrest and is discussed in more detail below. Overall, 21/24 $(87.5 \%)$ patients discontinued during the vaccine induction or consolidation phase (i.e., within the first 10 weeks of the study). Fourteen patients entered the consolidation phase, and three continued to the maintenance phase.

\subsection{Safety}

No DLT was observed in this study, and no patient discontinued DSP-7888 due to a TEAE. All patients experienced one or more TEAE (Table 2; Supplementary Table S3, see ESM), the most frequent of which was injection-site reaction (ISR). ISRs is a grouping comprising the following 
Table 1 Baseline demographics and disease characteristics

\begin{tabular}{|c|c|c|c|c|c|c|}
\hline & \multicolumn{3}{|l|}{$\begin{array}{l}\text { ID } \\
(n=10)\end{array}$} & \multicolumn{2}{|l|}{$\begin{array}{l}\mathrm{SC} \\
(n=14)\end{array}$} & \multirow[t]{2}{*}{$\begin{array}{l}\text { Overall } \\
(N=24)\end{array}$} \\
\hline & $\begin{array}{l}\text { DSP-7888 } 3.5 \mathrm{mg} \\
(n=4)\end{array}$ & $\begin{array}{l}\text { DSP-7888 } 10.5 \mathrm{mg} \\
(n=3)\end{array}$ & $\begin{array}{l}\text { DSP-7888 } 17.5 \mathrm{mg} \\
(n=3)\end{array}$ & $\begin{array}{l}\text { DSP-7888 } 3.5 \mathrm{mg} \\
(n=9)\end{array}$ & $\begin{array}{l}\text { DSP-7888 } 10.5 \mathrm{mg} \\
(n=5)\end{array}$ & \\
\hline $\begin{array}{l}\text { Median age, years } \\
\text { (range) }\end{array}$ & $61.0(53-67)$ & $53.0(51-70)$ & $67.0(64-73)$ & $66.0(43-70)$ & $44.0(23-84)$ & $63.0(23-84)$ \\
\hline Male, $n(\%)$ & $2(50.0)$ & $2(66.7)$ & $2(66.7)$ & $6(66.7)$ & $3(60.0)$ & $15(62.5)$ \\
\hline White, $n(\%)$ & $4(100)$ & $3(100)$ & $2(66.7)$ & $8(88.9)$ & $5(100)$ & $22(91.7)$ \\
\hline $\begin{array}{l}\text { Median BMI, kg/ } \\
\mathrm{m}^{2} \text { (range) }\end{array}$ & $\begin{array}{l}23.040(19.13- \\
27.77)\end{array}$ & $\begin{array}{l}30.394(21.38- \\
34.33)\end{array}$ & $\begin{array}{l}28.636(27.90- \\
45.94)\end{array}$ & $\begin{array}{l}27.840(20.20- \\
30.84)\end{array}$ & $\begin{array}{l}25.560(21.91- \\
28.89)\end{array}$ & $\begin{array}{l}27.617(19.13- \\
45.94)\end{array}$ \\
\hline \multicolumn{7}{|c|}{ Primary cancer type, $n(\%)$} \\
\hline GBM & $1(25.0)$ & 0 & 0 & $4(44.4)$ & $2(40.0)$ & $7(29.2)$ \\
\hline Pancreatic cancer & $2(50.0)$ & $1(33.3)$ & 0 & $2(22.2)$ & $2(40.0)$ & $7(29.2)$ \\
\hline Sarcoma & 0 & $2(66.7)$ & 0 & 0 & $1(20.0)$ & $3(12.5)$ \\
\hline MDS & 0 & 0 & $2(66.7)$ & 0 & 0 & $2(8.3)$ \\
\hline NSCLC & 0 & 0 & $1(33.3)$ & $1(11.1)$ & 0 & $2(8.3)$ \\
\hline Ovarian cancer & $1(25.0)$ & 0 & 0 & $1(11.1)$ & 0 & $2(8.3)$ \\
\hline Melanoma & 0 & 0 & 0 & $1(11.1)$ & 0 & $1(4.2)$ \\
\hline \multicolumn{7}{|c|}{ Type of prior cancer therapy, ${ }^{\mathrm{a}} n(\%)$} \\
\hline Chemotherapy & $4(100)$ & $2(66.7)$ & $3(100)$ & $9(100)$ & $5(100)$ & $23(95.8)$ \\
\hline Biologic therapy & $1(25.0)$ & 0 & 0 & $2(22.2)$ & $1(20.0)$ & $4(16.7)$ \\
\hline Immunotherapy & $1(25.0)$ & 0 & $1(33.3)$ & $5(55.6)$ & $2(40.0)$ & $9(37.5)$ \\
\hline $\begin{array}{l}\text { Investigational } \\
\text { agent }\end{array}$ & $1(25.0)$ & 0 & $1(33.3)$ & $4(44.4)$ & $1(20.0)$ & $7(29.2)$ \\
\hline \multicolumn{7}{|c|}{ Best response to prior cancer therapy, $n(\%)$} \\
\hline $\mathrm{CR}$ & 0 & $1(33.3)$ & 0 & 0 & 0 & $1(4.2)$ \\
\hline PR & $1(25.0)$ & 0 & 0 & $1(11.1)$ & 0 & $2(8.3)$ \\
\hline SD & $1(25.0)$ & $1(33.3)$ & $3(100)$ & $5(55.6)$ & $4(80.0)$ & $14(58.3)$ \\
\hline PD & $2(50.0)$ & 0 & 0 & $3(33.3)$ & $1(20.0)$ & $6(25.0)$ \\
\hline \multicolumn{7}{|l|}{ HLA type, $n(\%)$} \\
\hline HLA-A*02:01 & $3(75.0)$ & $3(100)$ & $3(100)$ & $8(88.9)^{\mathrm{b}}$ & $3(60.0)^{\mathrm{b}}$ & $20(83.3)^{b}$ \\
\hline HLA-A*02:06 & 0 & 0 & 0 & 0 & 0 & 0 \\
\hline HLA-A*24:02 & $1(25.0)$ & 0 & 0 & $3(33.3)^{\mathrm{b}}$ & $3(60.0)^{b}$ & $7(29.2)^{\mathrm{b}}$ \\
\hline
\end{tabular}

$B M I$ body mass index, $C R$ complete response, GBM glioblastoma multiforme, $H L A$ human leukocyte antigen, $I D$ intradermal, $M D S$ myelodysplastic syndrome, $N S C L C$ non-small cell lung cancer, $P D$ progressive disease, $P R$ partial response, $S C$ subcutaneous, $S D$ stable disease

${ }^{a}$ Patients may have received multiple lines of previous therapy

${ }^{\mathrm{b}}$ Two patients in the SC DSP-7888 3.5-mg group and one patient in the SC DSP-7888 10.5-mg group had both HLA-A*02:01 and HLAA*24:02

preferred terms: ISR, injection-site erythema, injectionsite pain, injection-site pruritus, injection-site hypertrophy, and injection-site induration. All (100\% [10/10]) patients administered ID DSP-7888 experienced one or more ISR (grouping), with the most commonly reported preferred terms being ISR $(90.0 \%$ [9/10]) and injection-site pruritus $(20.0 \%$ [2/10]). In total, $35.7 \%$ (5/14) of patients administered SC DSP-7888 experienced one or more ISR (grouping), most commonly injection-site erythema (21.4\% [3/14]) and injection-site pain $(14.3 \%$ [2/14]). All reports of ISRs with both formulations of DSP-7888 were grade 1 or 2 and did not lead to any treatment discontinuation.
Overall, four (40\%) patients in the ID cohort and five $(35.7 \%)$ in the SC cohort experienced grade $\geq 3$ TEAEs. There was one grade 4 TEAE (jaundice), which was reported in a patient with pancreatic cancer and hepatomegaly with liver metastasis who also presented with hyperbilirubinemia (grade 3; serious TEAE), which resolved within 3 days of onset and was deemed by the investigator to be related to disease progression. A greater percentage of patients experienced treatment-related TEAEs with ID versus SC administration of DSP-7888 (100\% [10/10] versus 50.0\% [7/14]), which was primarily attributable to ISRs (100\% [10/10] versus $35.7 \%$ [5/14]). Of the 15 patients who had an ISR, 
six were treated with a dermal steroid treatment (ID, $40.0 \%$ [4/10]; SC, 40.0\% [2/5]) and nine received no treatment (ID, $60.0 \%$ [6/10]; SC, 60\% [3/5]). No patient in either the SC or ID cohort who had an ISR required a dose interruption.

Five serious TEAEs were reported in four patients (ID $3.5 \mathrm{mg}$ : hyperbilirubinemia [discussed above]; ID $17.5 \mathrm{mg}$ : atrial fibrillation and anemia; SC $3.5 \mathrm{mg}$ : cardio-pulmonary arrest; SC $10.5 \mathrm{mg}$ : death due to disease progression). None was considered by the investigator to be related to DSP7888. The two patients with serious TEAEs in the ID cohorts recovered, whereas both patients with serious TEAEs in the SC cohorts died. The two serious TEAEs leading to death were cardio-respiratory arrest in a patient with metastatic NSCLC and disease progression in a patient with metastatic pancreatic cancer. Neither death was deemed by the investigator to be related to study treatment.

There was no increase in TEAE frequency with increasing DSP-7888 dose, irrespective of formulation. No formal RP2D was established in this study.

\subsection{Anti-Tumor Activity}

Four (16.7\%) patients had stable disease (SD; ID $17.5 \mathrm{mg}$ [MDS], $n=1$; SC $3.5 \mathrm{mg}, n=1$ [melanoma]; SC $10.5 \mathrm{mg}$, $n=2$ [glioblastoma multiforme, sarcoma]), $16(66.7 \%)$ had progressive disease (PD), and 4 (16.7\%) were not evaluable for tumor response (Supplementary Figure S2, see ESM). Of the 18 patients with advanced solid tumors, 15 had a best overall response (BOR) of PD and three had a BOR of SD. Of the two patients with MDS, one had a BOR of SD and one had a BOR of PD. No patient had a complete or partial response. By end of study, 14 (58.3\%) patients had died. Median (95\% confidence interval $[\mathrm{CI}])$ OS was 180.0 (136.0-494.0) days, ranging from $103.0(42.0-180.0)$ to 311.0 (97.0-311.0) days across all five dosing cohorts (Table 3). Median (95\% CI) PFS was $52.0(41.0-57.0)$ days, ranging from 48.5 (12.0-57.0) to 77.0 (33.0-103.0) days across all five dosing cohorts (see Table 3).

No patient presented initially with AML. Two patients with MDS were enrolled; both were assigned to ID DSP-7888 $17.5 \mathrm{mg}$, and both experienced transformation to AML. Median (95\% CI) time from first dose of DSP-7888 to transformation to AML was 305.5 (117.0-494.0) days. Neither patient was in transformation prior to the administration of DSP-7888, but at the time of enrollment both were considered high risk for AML per IPSS. One of these patients had discontinued the study due to disease progression and the other had withdrawn with SD.

\subsection{Pharmacodynamics}

To evaluate the immune response following administration of DSP-7888, WT1-specific CTL induction activity was measured in peripheral whole blood. A total of 21 patients were evaluable for the assessment of WT1-specific CTLs (ID, $n=9$; SC, $n=12$ ). Although dose-dependent WT1-specific CTL induction activity was not observed, higher WT1-specific CTL induction was observed in the ID group than in the SC group (66.7\% [6/9] vs 41.7\% [5/12] of patients) (Supplementary Tables S2 and S3, see ESM).

HLA expression was evaluated to determine whether the assessed cancers evaded immunotherapy with DSP-7888 (i.e., loss of HLA expression would indicate immune evasion). Seventeen patients with solid tumors provided biopsies for the assessment of HLA class I membrane expression; two were not evaluable due to no pretreatment sample collection. Of the 15 evaluable patients, HLA class I membrane expression was generally strong, with $11(73.3 \%)$ tumors graded as $3+(\geq 90 \%$ cells with moderate or greater stain) and $4(26.7 \%)$ tumors graded as $1+(10 \%$ to $<50 \%$ cells with moderate or greater stain, or $\geq 10 \%$ cells with weak stain). Sixteen patients provided biopsies for the assessment of HLA class II membrane expression; one was not evaluable. Of the remaining 15 patients, HLA class II membrane expression was detected in $11(73.3 \%)$ and not detected in $4(26.7 \%)$.

\section{Discussion}

In this phase I study of patients with advanced malignancies associated with overexpression of WT1, both ID and SC formulations of DSP-7888 were found to be well tolerated, with no DLTs, dose reductions, or treatment discontinuations due to toxicity other than one death deemed not related to study drug. The most common TEAEs were ISRs (grouping), which were more common with ID versus SC administration of DSP-7888 (100\% versus 35.7\%). ISR was the most frequently reported preferred term among patients administered ID DSP-7888, whereas injection-site erythema was the most frequently reported preferred term among those administered SC DSP-7888. No patient discontinued or required a dose interruption due to an ISR, which was effectively managed with minimal intervention. Of the 15 patients who had an ISR, six received topical steroid treatment, whereas nine patients required no treatment.

Efforts to target WT1 as a potential cancer therapy have utilized various approaches, including $\mathrm{T}$ cell receptor gene therapy, dendritic cells, and plasmids [33-37]. Peptide-based vaccines targeting WT1 are the most relevant to the current study and have been shown to elicit the production of WT1specific CTLs [18-21, 25, 38]. In contrast with previously studied WT1 vaccines, DSP-7888 is expected to stimulate both WT1-specific CTLs and HTLs, rather than CTLs or HTLs alone [22, 23, 39]. The exclusive targeting of WT1specific CTLs by previously studied WT1 vaccines had only minimal clinical effect in patients with solid tumors [19, 20,40]. HTLs function in anti-cancer responses by priming and recruiting CTLs and activating CTL memory responses, 


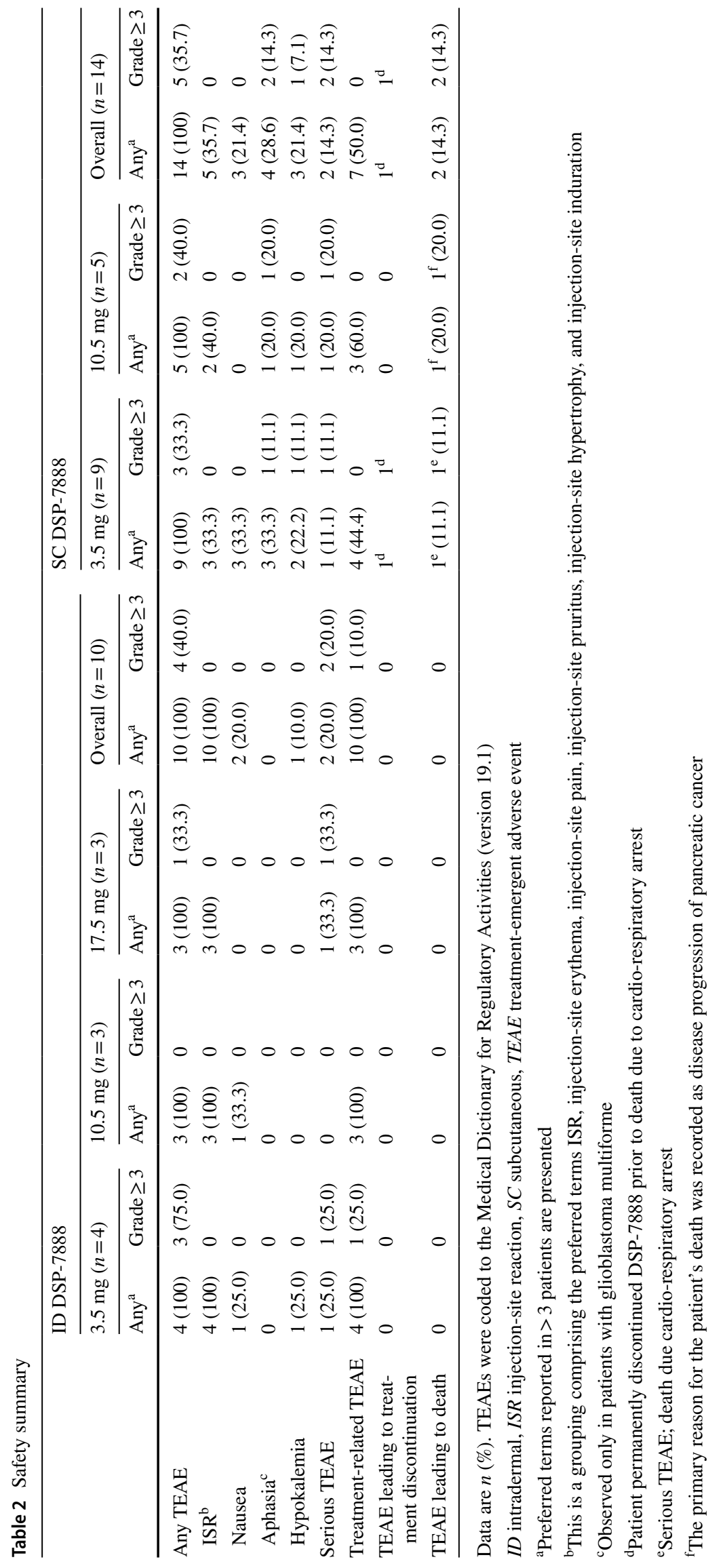


Table 3 Median OS (intent-to-treat) and PFS (intent-to-treat)

\begin{tabular}{|c|c|c|c|c|c|c|}
\hline & \multicolumn{3}{|l|}{$\begin{array}{l}\text { ID DSP-7888 } \\
n=10\end{array}$} & \multicolumn{2}{|l|}{$\begin{array}{l}\text { SC DSP-7888 } \\
n=14\end{array}$} & \multirow[t]{2}{*}{$\begin{array}{l}\text { Overall } \\
(N=24)\end{array}$} \\
\hline & $\begin{array}{l}3.5 \mathrm{mg} \\
(n=4)\end{array}$ & $\begin{array}{l}10.5 \mathrm{mg} \\
(n=3)\end{array}$ & $\begin{array}{l}17.5 \mathrm{mg} \\
(n=3)\end{array}$ & $\begin{array}{l}3.5 \mathrm{mg} \\
(n=9)\end{array}$ & $\begin{array}{l}10.5 \mathrm{mg} \\
(n=5)\end{array}$ & \\
\hline $\begin{array}{l}\text { Median OS, days } \\
\quad(95 \% \text { CI })\end{array}$ & $176.0(\mathrm{NE}-\mathrm{NE})$ & NE (182.0-NE) & $220.0(117.0-494.0)$ & $311.0(97.0-311.0)$ & $103.0(42.0-180.0)$ & $180.0(136.0-494.0)$ \\
\hline $\begin{array}{l}\text { Median PFS, days } \\
\quad(95 \% \mathrm{CI})\end{array}$ & $51.0(33.0-56.0$ & $62.0(52.0-66.0)$ & $54.0(29.0-\mathrm{NE})$ & $48.5(12.0-57.0)$ & $77.0(33.0-103.0)$ & $52.0(41.0-57.0)$ \\
\hline
\end{tabular}

$C I$ confidence interval, $I D$ intradermal, $N E$ not evaluable, $O S$ overall survival, $P F S$ progression-free survival, $S C$ subcutaneous

so optimal anti-tumor responses against WT1 likely require both WT1-specific CTLs and HTLs [22, 23, 39], which DSP7888 is expected to achieve (Sumitomo Dainippon Pharma Oncology, Inc., data on file). Of note, in the current study, DSP-7888 induced WT1-specific CTLs that were measurable without the need for amplification via ex vivo culture. WT1-specific CTLs were induced in $66.7 \%$ (6/9) of patients administered ID DSP-7888 compared with $56.5 \%$ (13/23) of patients administered an ID WT1 vaccine designed primarily to elicit WT1-specific CTLs [18]. SC-administered DSP7888 resulted in lower WT1-specific CTL induction $(41.7 \%$ [5/12 patients]) than ID-administered DSP-7888 (Supplementary Table S3, see ESM). However, the sample sizes in these studies were small, so future assessment of larger patient populations will better inform the utility of stimulating both CTL and HTL responses. The safety profile of DSP-7888 is comparable with other WT1-targeted peptidebased vaccines, with patients experiencing ISRs that were most commonly grade 1-2 injection-site erythema [18-21, $25,38]$.

Across all tumor types and dose cohorts, OS duration was limited, which is not unexpected in a heavily pretreated advanced cancer patient population. Tumor responses were unlikely to have been influenced by either the dosing route (ID versus SC) or dose of DSP-7888 administered, although patient numbers in each cohort were small. As data emerged from the study suggesting that WT1-specific CTL induction with DSP-7888 was higher with ID administration compared with $\mathrm{SC}$, the study evaluated dose levels from $3.5 \mathrm{mg}$ to $17.5 \mathrm{mg}$ with ID administration. However, assessment of the 17.5-mg dose given SC was not deemed necessary as lower CTL induction was expected from this route of administration.

No formal RP2D was determined in the present study; DSP-7888 Dosing Emulsion was well tolerated, with no DLTs observed. However, based on a comprehensive review of safety, tolerability, and biomarker information from each cohort in this study and other available clinical trial data, DSP-7888 $10.5 \mathrm{mg}$ ID administration has been selected for further clinical evaluation in adult patients with malignancies for the following reasons: (i) the density of dendritic cells in ID compared with
SC sites facilitates the capture of tumor antigens, leading to enhanced immune responses; thus, the dermis is a preferred site of administration for tumor vaccines [41]; (ii) higher WT1specific CTLs induction activity was noted with ID versus SC administration; (iii) it was a well-tolerated and safe dose; and (iv) fewer injection sites are required compared with the highest dose tested (17.5 mg ID) (Supplementary Table S3, see ESM).

\section{Conclusions}

In this study, DSP-7888 Dosing Emulsion was well tolerated and safe, with no dose-limiting toxicities, in patients with recurrent or advanced malignancies. Higher WT1-specific CTL induction activity was noted with ID compared with $\mathrm{SC}$ administration, and the ID route was selected for further evaluation in the clinical program.

Supplementary Information The online version contains supplementary material available at https://doi.org/10.1007/s11523-021-00813-6.

Acknowledgements Medical writing assistance was provided by Christina Khodr, PhD and Rob Steger, PhD of Ashfield MedComms, an Ashfield Health Company, and was funded by Sumitomo Dainippon Pharma Oncology, Inc.

\section{Declarations}

Funding This work was supported by Sumitomo Dainippon Pharma Oncology, Inc.

Competing interests AS has received research support from Boston Biomedical, Inc. (now Sumitomo Dainippon Pharma Oncology, Inc.) and has consulted for AstraZeneca, Bristol-Myers Squibb, Novartis, and Takeda. ARH reports consulting or an advisory role for Merck, GlaxoSmithKline, Bristol-Myers Squibb, and Eisai, and research funding (paid to institution) from Karyopharm Therapeutics, Merck, Bristol-Meyers Squibb, GlaxoSmithKline, Roche/Genentech, Janssen, AstraZeneca/Medimmune, Astellas Pharma, Macrogenics, and Boehringer Ingelheim.. WAH has nothing to disclose. KKC is an employee of Syneos Health. EK-Y is an employee of Sumitomo Dainippon Pharma Co., Ltd (parent company of Sumitomo Dainippon Pharma Oncology, Inc.). MO and ZL are employees of Sumitomo Dainippon Pharma Oncology, Inc. BE is a former employee of Boston Biomedical, 
Inc (now Sumitomo Dainippon Pharma Oncology, Inc.). WLS received research support from Boston Biomedical, Inc. (now Sumitomo Dainippon Pharma Oncology, Inc.), Eli Lily and Company, and Lexicon Pharmaceuticals; and honoraria for consultation from Blueprint Medicines, Ipsen, Lexicon Pharmaceuticals, Mylan, and Natera, Inc.

Ethics approval This study was conducted in compliance with the Declaration of Helsinki, the International Conference on Harmonization Guidelines for Good Clinical Practice, and applicable national and local regulatory requirements. The study protocol was approved by the Independent Ethics Committee or Institutional Review Board at each participating site: the University Health Network Research Ethics Board (FWA00000518); Emory University Institutional Review Board (FWA00005792_IRB00082294); Western Institutional Review Board (IRB00000533_IORG0000432); Yale University Human Subjects Committee (FWA00002571); Cleveland Clinic Institutional Review Board (FWA00005367); USOncology Inc. Institutional Review Board (IORG0000759).

Consent to participate All patients provided written informed consent prior to study participation.

\section{Consent for publication Not applicable.}

Availability of data and material The datasets used and/or analyzed during the current study are available from the corresponding author on reasonable request.

\section{Code availability Not applicable.}

Authors' contributions AS, ARH, WAH, KKC, and WLS treated patients and performed the clinical data acquisition. ZL provided statistical data analysis and $\mathrm{KKC}, \mathrm{EK}-\mathrm{Y}, \mathrm{MO}$, and $\mathrm{BE}$ interpreted the data. All authors contributed to writing and critically revising the manuscript for important intellectual content and approved the final draft. All authors agree to be accountable for all aspects of the work in ensuring that questions related to the accuracy or integrity of any part of the work are appropriately investigated and resolved.

Open Access This article is licensed under a Creative Commons Attribution-NonCommercial 4.0 International License, which permits any non-commercial use, sharing, adaptation, distribution and reproduction in any medium or format, as long as you give appropriate credit to the original author(s) and the source, provide a link to the Creative Commons licence, and indicate if changes were made. The images or other third party material in this article are included in the article's Creative Commons licence, unless indicated otherwise in a credit line to the material. If material is not included in the article's Creative Commons licence and your intended use is not permitted by statutory regulation or exceeds the permitted use, you will need to obtain permission directly from the copyright holder. To view a copy of this licence, visit http://creativecommons.org/licenses/by-nc/4.0/.

\section{References}

1. Loeb DM, Evron E, Patel CB, Sharma PM, Niranjan B, Buluwela L, et al. Wilms' tumor suppressor gene (WT1) is expressed in primary breast tumors despite tumor-specific promoter methylation. Cancer Res. 2001;61:921-5.

2. Oji Y, Miyoshi S, Maeda H, Hayashi S, Tamaki H, Nakatsuka SI, et al. Overexpression of the Wilms' tumor gene WT1 in de novo lung cancers. Int J Cancer. 2002;100:297-303.
3. Oji Y, Yamamoto H, Nomura M, Nakano Y, Ikeba A, Nakatsuka SI, et al. Overexpression of the Wilms' tumor gene WT1 in colorectal adenocarcinoma. Cancer Sci. 2003;94:712-7.

4. Oji Y, Nakamori S, Fujikawa M, Nakatsuka SI, Yokota A, Tatsumi N, et al. Overexpression of the Wilms' tumor gene WT1 in pancreatic ductal adenocarcinoma. Cancer Sci. 2004;95:583-7.

5. Nakahara Y, Okamoto H, Mineta T, Tabuchi K. Expression of the Wilms' tumor gene product WT1 in glioblastomas and medulloblastomas. Brain Tumor Pathol. 2004;21:113-6.

6. Inoue $\mathrm{K}$, Sugiyama $\mathrm{H}$, Ogawa $\mathrm{H}$, Nakagawa $\mathrm{M}$, Yamagami $\mathrm{T}$, Miwa $\mathrm{H}$, et al. WT1 as a new prognostic factor and a new marker for the detection of minimal residual disease in acute leukemia. Blood. 1994;84:3071-9.

7. Qi XW, Zhang F, Wu H, Liu JI, Zong B, Xu C, et al. Wilms' tumor 1 (WT1) expression and prognosis in solid cancer patients: a systematic review and meta-analysis. Sci Rep. 2015;5:8924.

8. Østergaard M, Olesen LH, Hasle H, Kjeldsen E, Hokland P. WT1 gene expression: an excellent tool for monitoring minimal residual disease in $70 \%$ of acute myeloid leukaemia patients-results from a single-centre study. Br J Haematol. 2004;125:590-600.

9. Cilloni D, Renneville A, Hermitte F, Hills RK, Daly S, Jovanovic $\mathrm{JV}$, et al. Real-time quantitative polymerase chain reaction detection of minimal residual disease by standardized WT1 assay to enhance risk stratification in acute myeloid leukemia: a European LeukemiaNet study. J Clin Oncol. 2009;27:5195-201.

10. Rosenfeld C, Cheever MA, Gaiger A. WT1 in acute leukemia, chronic myelogenous leukemia and myelodysplastic syndrome: therapeutic potential of WT1 targeted therapies. Leukemia. 2003;17:1301-12.

11. Miyagawa K, Kent J, Schedl A, van Heyningen V, Hastie ND. Wilms' tumour-a case of disrupted development. J Cell Sci Suppl. 1994;18:1-5.

12. Inoue $\mathrm{K}$, Ogawa $\mathrm{H}$, Yamagami $\mathrm{T}$, Soma $\mathrm{T}$, Tani $\mathrm{Y}$, Tatekawa $\mathrm{T}$, et al. Long-term follow-up of minimal residual disease in leukemia patients by monitoring WT1 (Wilms tumor gene) expression levels. Blood. 1996;88:2267-78.

13. Tamaki H, Ogawa H, Inoue K, Soma T, Yamagami T, Miyake $\mathrm{S}$, et al. Increased expression of the Wilms tumor gene (WT1) at relapse in acute leukemia. Blood. 1996;88:4396-8.

14. Miyoshi Y, Ando A, Egawa C, Taguchi T, Tamaki Y, Tamaki $\mathrm{H}$, et al. High expression of Wilms' tumor suppressor gene predicts poor prognosis in breast cancer patients. Clin Cancer Res. 2002;8:1167-71.

15. Oji Y, Ogawa H, Tamaki H, Oka Y, Tsuboi A, Kim EH, et al. Expression of the Wilms' tumor gene WT1 in solid tumors and its involvement in tumor cell growth. Jpn J Cancer Res. 1999;90:194-204.

16. Yamagami T, Sugiyama H, Inoue K, Ogawa H, Tatewaka T, Hirata $\mathrm{M}$, et al. Growth inhibition of human leukemic cells by WT1 (Wilms tumor gene) antisense oligodeoxynucleotides: implications for the involvement of WT1 in leukemogenesis. Blood. 1996;87:2878-84.

17. Algar EM, Khromykh T, Smith SI, Blackburn DM, Bryson GJ, Smith PJ. A WT1 antisense oligonucleotide inhibits proliferation and induces apoptosis in myeloid leukaemia cell lines. Oncogene. 1996;12:1005-14.

18. Oka Y, Tsuboi A, Taguchi T, Osaki T, Kyo T, Nakajima H, et al. Induction of WT1 (Wilms' tumor gene)-specific cytotoxic T lymphocytes by WT1 peptide vaccine and the resultant cancer regression. Proc Natl Acad Sci USA. 2004;101:13885-90.

19. Morita S, Oka Y, Tsuboi A, Kawakami M, Maruno M, Izumoto $\mathrm{S}$, et al. A phase I/II trial of a WT1 (Wilms' tumor gene) peptide vaccine in patients with solid malignancy: safety assessment based on the phase I data. Jpn J Clin Oncol. 2006;36:231-6.

20. Izumoto S, Tsuboi A, Oka Y, Suzuki T, Hashiba T, Kagawa N, et al. Phase II clinical trial of Wilms tumor 1 peptide vaccination 
for patients with recurrent glioblastoma multiforme. J Neurosurg. 2008;108:963-71.

21. Ohno S, Kyo S, Myojo S, Dohi S, Ishizaki J, Miyamoto KI, et al. Wilms' tumor 1 (WT1) peptide immunotherapy for gynecological malignancy. Anticancer Res. 2009;29:4779-84.

22. Hung K, Hayashi R, Lafond-Walker A, Lowenstein C, Pardoll D, Levitsky H. The central role of CD4(+) T cells in the antitumor immune response. J Exp Med. 1998;188:2357-68.

23. Wang RF. The role of MHC class II-restricted tumor antigens and CD4+ T cells in antitumor immunity. Trends Immunol. 2001;22:269-76.

24. Janssen EM, Lemmens EE, Wolfe T, Christen U, van Herrath MG, Schoenberger SP. CD4+ T cells are required for secondary expansion and memory in CD8+ T lymphocytes. Nature. 2003;421:852-6.

25. Kobayashi Y, Sakura T, Miyawaki S, Toga K, Sogo S, Heike Y. A new peptide vaccine OCV-501: in vitro pharmacology and phase 1 study in patients with acute myeloid leukemia. Cancer Immunol Immunother. 2017;66:851-63.

26. Tsuboi A, Oka Y, Udaka K, Murakami M, Masuda T, Nakano A, et al. Enhanced induction of human WT1-specific cytotoxic T lymphocytes with a 9-mer WT1 peptide modified at HLA-A*2402-binding residues. Cancer Immunol Immunother. 2002;51:614-20.

27. Pinilla-Ibarz J, May RJ, Korontsvit T, Gomez M, Kappel B, Zakhaleva $\mathrm{V}$, et al. Improved human T-cell responses against synthetic HLA-0201 analog peptides derived from the WT1 oncoprotein. Leukemia. 2006;20:2025-33.

28. Wolchok JD, Hoos A, O'Day S, Weber JS, Hamid O, Lebbé C, et al. Guidelines for the evaluation of immune therapy activity in solid tumors: immune-related response criteria. Clin Cancer Res. 2009;15:7412-20.

29. Hoos A, Eggermont AM, Janetzki S, Hodi FS, Ibrahim R, Anderson A, et al. Improved endpoints for cancer immunotherapy trials. J Natl Cancer Inst. 2010;102:1388-97.

30. Rustin GJ, Vergote I, Eisenhauer E, Pujade-Lauraine E, Quinn $\mathrm{M}$, Thigpen T, et al. Definitions for response and progression in ovarian cancer clinical trials incorporating RECIST 1.1 and CA 125 agreed by the Gynecological Cancer Intergroup (GCIG). Int J Gynecol Cancer. 2011;21:419-23.

31. Cheson BD, Bennett JM, Kopecky KJ, Büchner T, Willman CL, Estey EH, et al. Revised recommendations of the International Working Group for Diagnosis, Standardization of Response Criteria, Treatment Outcomes, and Reporting Standards for
Therapeutic Trials in Acute Myeloid Leukemia. J Clin Oncol. 2003;21:4642-9.

32. Cheson BD, Greenberg PL, Bennett JM, Lowenberg B, Wijermans PW, Nimer SD, et al. Clinical application and proposal for modification of the International Working Group (IWG) response criteria in myelodysplasia. Blood. 2006;108:419-25.

33. Chapuis AG, Egan DN, Bar M, Schmitt TM, McAfee MS, Paulson $\mathrm{KG}$, et al. T cell receptor gene therapy targeting WT1 prevents acute myeloid leukemia relapse post-transplant. Nat Med. 2019;25:1064-72.

34. Katsuda M, Miyazawa M, Ojima T, Katanuma A, Hakamada K, Sudo K, et al. A double-blind randomized comparative clinical trial to evaluate the safety and efficacy of dendritic cell vaccine loaded with WT1 peptides (TLP0-001) in combination with S-1 in patients with advanced pancreatic cancer refractory to standard chemotherapy. Trials. 2019;20(1):242.

35. Eckl J, Raffegerst S, Schnorfeil F, Prinz-Schulz P, Fingerhut $\mathrm{C}$, Floisand $\mathrm{Y}$, et al. DC Vaccination induces antigen specific immune responses in AML patients: a 1-year interim assessment. Blood. 2019;134(Suppl 1):3923.

36. ClinicalTrials.gov identifier: NCT03491683. https://clinicaltrials. gov/ct2/show/NCT03491683.

37. Reardon DA, Brem S, Desai AS, Bagley SJ, Kurz SC, De La Fuente MI, et al. INO-5401 and INO-9012 delivered intramuscularly (IM) with electroporation (EP) in combination with cemiplimab (REGN2810) in newly diagnosed glioblastoma (GBM): interim results. J Clin Oncol. 2020;38(Suppl 15):2514.

38. Maslak PG, Dao T, Bernal Y, Chanel SM, Zhang R, Frattini $\mathrm{M}$, et al. Phase 2 trial of a multivalent WT1 peptide vaccine (galinpepimut-S) in acute myeloid leukemia. Blood Adv. 2018;2(3):224-34.

39. Goto M, Nakamura M, Suginobe N, Takasu H, Takanashi Y, Ban $\mathrm{H}$, et al. DSP-7888, a novel cocktail design of WT1 peptide vaccine, and its combinational immunotherapy with immune checkpoint-blocking antibody against PD-1. Blood. 2016;128:4715.

40. Fu S, Piccioni DE, Liu H, Lukas RV, Aregawi D, Yamaguchi $\mathrm{K}$, et al. Initial phase 1 study of WT2725 dosing emulsion in patients with advanced malignancies. J Clin Oncol. 2017;35(Suppl 15):2066.

41. Bonnotte B, Gough M, Phan V, Ahmed A, Chong H, Martin F, et al. Intradermal injection, as opposed to subcutaneous injection, enhances immunogenicity and suppresses tumorigenicity of tumor cells. Cancer Res. 2003;63:2145-9.

\section{Authors and Affiliations}

\section{Alexander Spira ${ }^{1,2}$ (1) $\cdot$ Aaron R. Hansen ${ }^{3} \cdot$ Wael A. Harb ${ }^{4} \cdot$ Kelly K. Curtis $^{5} \cdot$ Erina Koga-Yamakawa $^{6}$. Makoto Origuchi ${ }^{7} \cdot$ Zhonggai Li $^{8} \cdot$ Bella Ertik ${ }^{9}$. Walid L. Shaib ${ }^{10}$}

$1 \quad$ Virginia Cancer Specialists, 8503 Arlington Blvd., Suite 400, Fairfax, VA 22031, USA

2 The US Oncology Network, The Woodlands, TX, USA

3 Division of Medical Oncology and Hematology, Princess Margaret Cancer Centre, Toronto, ON, Canada

4 Horizon Oncology Research, LLC, Lafayette, IN, USA

5 Medical Management and Scientific Services, Syneos Health, Phoenix, AZ, USA

6 DSP Cancer Institute, Sumitomo Dainippon Pharma Co., Ltd, Osaka, Japan
7 Clinical Development, Sumitomo Dainippon Pharma Oncology, Inc., Cambridge, MA, USA

8 Biostatistics, Sumitomo Dainippon Pharma Oncology, Inc., Cambridge, MA, USA

9 Pharmcovigilance, Former Employee of Boston Biomedical, Inc. (Now Sumitomo Dainippon Pharma Oncology, Inc.), Cambridge, MA, USA

10 Department of Hematology and Oncology, Winship Cancer Institute, Emory University, Atlanta, GA, USA 